\title{
Light and electron microscopic features of tropical ulcer
}

\author{
BEVERLEY ADRIAANS, R HAY, S LUCAS, * D C ROBINSON \\ From the Medical Microbiology Department, the *Wolfson Tropical Pathology Unit, London School of Hygiene \\ and Tropical Medicine
}

SUMMARY The histopathological features of 20 tropical ulcers with the electron microscopic findings on seven biopsy specimens are reported. The main findings were loss of epidermis associated with extensive dermal oedema, infiltration by polymorphonuclear leucocytes, and disruption of collagen bundles. The presence of micro-organisms at the site of tissue damage was shown and compared with the morphology of the organisms grown in culture. The most commonly identified bacteria were pleomorphic rods whose electron microscopic appearances accorded most closely with Fusobacteria grown in vitro. Spirochaetes, identified ultrastructurally as Treponema sp, were also present. There was no evidence of vasculitis to explain the rapid onset of ulceration, but necrotic changes seen in the dermis and the inflammatory cell infiltrate suggest that, associated with cell necrosis, bacteria previously shown in vitro have an important role in the pathogenesis of tropical ulcers.

Tropical ulcer predominantly affects children and is an acute inflammatory condition leading to skin necrosis. The lesions start from tiny papules which spread rapidly over a few days. ${ }^{1}$ Many patients give a history of preceding trauma, and it is believed that the lesion subsequently develops as a result of necrosis caused by a synergistic infection by one or more anaerobic bacteria such as Fusobacterium sp and aerobic organisms. These were recently isolated from lesions ${ }^{2}$ and had previously been shown in smears. ${ }^{3}$ The mechanisms of necrosis are not well understood and it is not known whether the organisms are present at the site of tissue damage. Necrosis seems to develop rapidly, which suggests that factors such as vascular damage may play a part in its pathogenesis.

We studied the light and electron microscopic features of patients with tropical ulcer in four endemic areas: Zambia, Gambia, southern India, and Papua New Guinea, to see whether the organisms isolated from ulcers are present at the site of pathological change and whether there was evidence of other potential sources of tissue damage such as vasculitis.

\section{Patients and methods}

Twenty patients with tropical ulcers were seen in clinics in the four endemic areas. The clinical features of the ulcers have been described elsewhere. ${ }^{4}$ The duration of disease and details of any treatment given were noted. Biopsy specimens were taken with a $4 \mathrm{~mm}$ disposable skin biopsy punch (Stiefel Laboratories, Maidenhead, United Kingdom) under local anaesthesia using $2 \%$ lignocaine with adrenalin across the edge of the ulcers. All specimens were fixed in $10 \%$ formol saline before being embedded in paraffin. Sections ( $5 \mu \mathrm{m}$ thick) were cut and stained with haematoxylin and eosin, Giemsa, Gram stain, ZiehlNeelsen, periodic acid Schiff, and by the Dieterle method for spirochaetes. All light microscopic biopsy specimens examined by light microscopy were evaluated and scored by three observers.

In seven cases fresh samples were divided and one half was processed for electron microscopy. The samples were fixed in $3 \%$ glutaraldehyde in $0.1 \mathrm{M}$ cacodylate buffer overnight. They were then fixed in $1 \%$ osmium tetroxide, $\mathrm{pH} 7 \cdot 2$, dehydrated through graded alcohols and embedded in epoxy resin, Epon 812. Semithin sections of $0.5 \mu \mathrm{m}$ were cut out on an ultramicrotome and treated with lead acetate. The 
sections were examined with an AEI 801 TEM at 60 and $80 \mathrm{kv}$ acceleration voltages and a $50 \mu \mathrm{m}$ aperture. For comparison, cultured samples of Fusobacteria, Bacteroides, and Enterobacter cloacae isolated from patients with tropical ulcer and identified by well established criteria, ${ }^{5}$ were also fixed as shown and examined.

\section{Results}

Twenty biopsy specimens were examined. In all cases the site of the lesion was the lower leg or foot. In 17 cases the duration of the ulcer was known and of these, nine had been present for less than one month, the rest were between four and eight weeks old. Seven had been treated with antibiotics (penicillin $n=6$, metronidazole $n=1$ ).

The light microscopic features could be divided into epidermal and subepidermal changes.

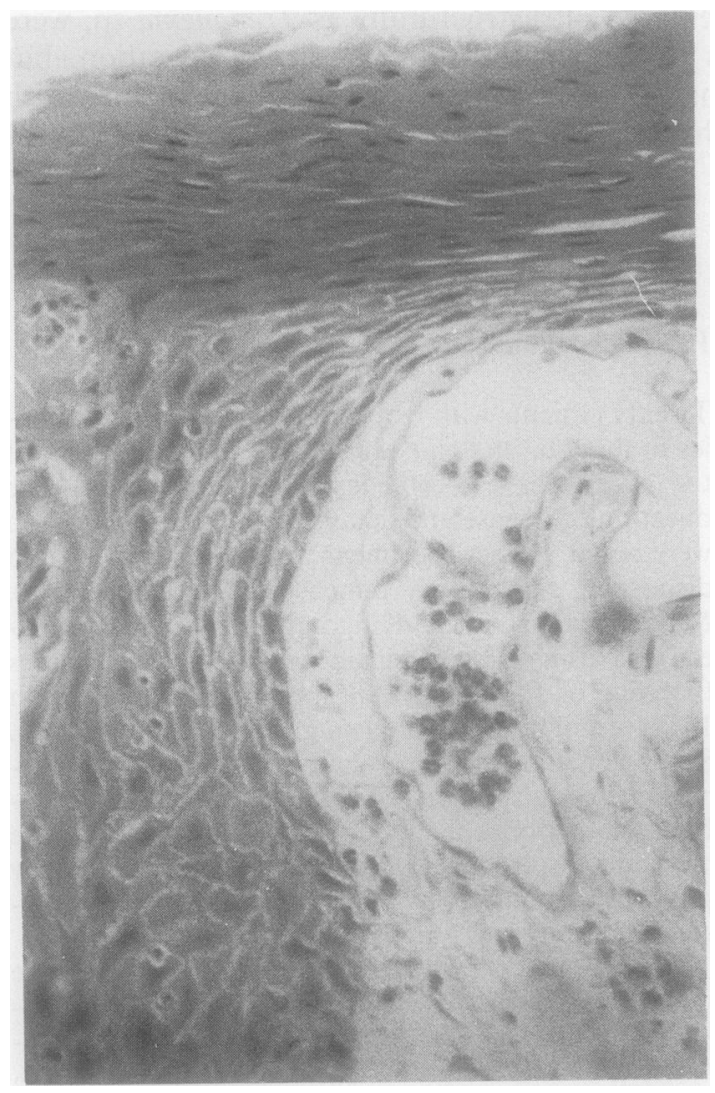

Fig 1 Edge of an acute tropical ulcer showing vascular dilatation, dermal oedema, and diffuse infiltration of epidermis with polymorphonuclear leucocytes (haematoxylin and eosin).
EPIDERMAL CHANGES

The main abnormality seen in all patients was the development of acanthosis around the ulcer rim, which was accompanied by a variable degree of hypkeratosis. In five patients who all reported ulcers of less than one month's duration, spongiosis was seen, which was accompanied by a diffuse infiltrate of mainly polymorphonuclear leucocytes in all layers of the epidermis (fig 1). Even at the edge of the ulcer, however, the basal epidermal layer appeared orderly and intact. In older lesions elongation of the rete ridges accompanied pronounced epidermal thickening. Remnants of stratum corneum were occasionally seen in the slough covering the ulcer.

Bacteria were seen in the epidermis in six patients, only one of whom had received antibiotics. All of these had ulcers of less than one month's duration. In four cases the organisms were seen in the outer stratum corneum, together with polymorphonuclear leucocytes. In two patients bacteria were seen scattered throughout all epidermal layers. For the most part, these were pleomorphic Gram negative rods or Gram positive cocci. In two patients spiral bacteria could be seen in the epidermal slough. The Dieterle stain proved very useful for showing all bacteria clearly. No fungi were identified in any of these sections.

\section{SUBEPIDERMAL CHANGES}

The pattern depended on the zone examined and the duration of the ulcers or treatment of the ulcers. The ulcer beds were unremarkable and showed granulation tissue with necrotic slough, polymorpho- $\frac{\alpha}{\Phi}$ nuclear leucocytes, and nuclear debris. At the base of $\stackrel{\varrho}{\Rightarrow}$ the ulcer vascular proliferation was commonly seen.

Under intact epidermis there was severe oedema of the dermis, particularly in ulcers less than one month old. This was absent in patients with ulcers of short duration who had received penicillin (fig 1). The cellu-용 lar infiltrate was dense and maximal around the epi- thelial defect. Most of the cells were polymorphonuclear leucocytes, although in two patients large numbers of eosinophils were seen. Lymphocytes were also present. Collagen bundles in the area of the ? infiltrate were widely separated, suggesting the pres- $\square$ ence of oedema. Next to the ulcers there was a dense음 inflammatory infiltrate around blood vessels and $\bar{N}$ adnexal structures. Nerves did not seem to be $\%$ affected. Pigmentary incontinence was seen in all N cases and usually extended well beyond the borders of the ulcer.

While the dermal blood vessels were dilated and

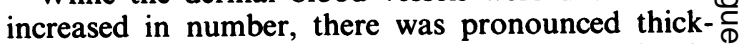
ening of vessel walls, with endothelial proliferation in $\stackrel{?}{?}$ several chronic ulcers. Dilatation without thickening $\square$ was mainly seen in acute lesions. There was no evi- 


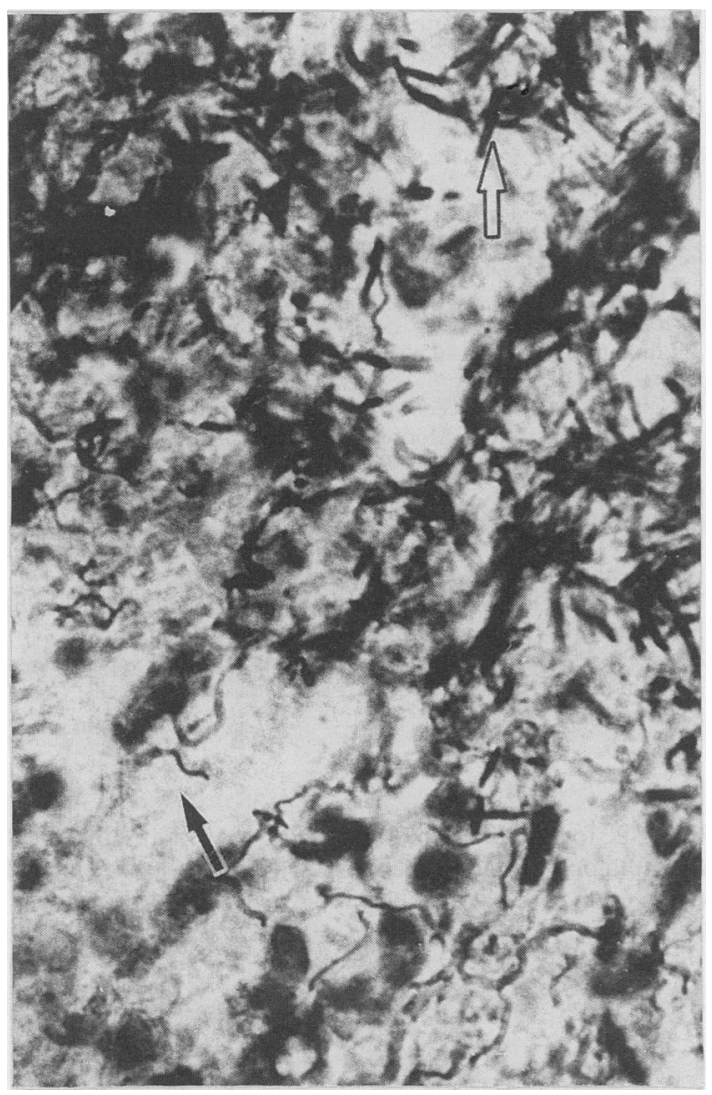

Fig 2 Pleomorphic bacterial rods and spirochaetes in an early untreated ulcer. (Dieterle stain.)

dence of vasculitis at any stage but one chronic ulcer showed endarteritis obliterans.

In acute lesions the sides and base of the ulcer were occupied by inflammatory slough containing bacteria. In untreated lesions these seemed to consist of a plug of necrotic material and organisms. The predominant bacteria were rod like or pleomorphic and were Gram negative. Long and arcuate forms were also seen (fig 2). In four untreated cases spiral bacteria were found in the slough or at the ulcer rim (fig 2). These were not associated with other organisms. Gram positive bacteria were rarely seen.

Sections from seven patients were examined by electron microscopy. The general features confirmed the light microscopic findings. In four acute cases the epidermal oedema was severe and the presence of polymorphonuclear leucocytes infiltrating the dermis was confirmed.

The predominating bacteria were rod shaped, round, or elongated with cytoplasm containing ribosomes and a thin cell wall, which tended to be irregular. The appearances accorded most with the fusobacterial isolates from culture. By contrast, organisms with the appearance of Bacteroides sp examined in vitro were only identified in two sections. These were rounder and had a more compact cytoplasm with thicker walls. The Coliform organisms were not seen in the tissue sections. Small cocci with dense staining cytoplasm were grouped around keratinocytes in some sections.

In two biopsy specimens spirochaetes were seen. These occurred in the deeper layers of the dermis and were not associated with other bacteria. The organisms showed sinusoidal periodicity and could be shown to have three flagellae. Details of the insertion of the flagellae could not be seen. These characteristics, however, corresponded with those of spirochaetes. ${ }^{6}$ Some pleomorphic bacteria were present in macrophages or polymorphonuclear leucocytes. From their appearance it seemed likely that they had resisted intracellular digestion. While it is difficult to comment on the clinical importance of the collagen changes, many disrupted bundles of collagen were seen in the vicinity of clusters of bacteria, suggesting that necrosis may have been induced by toxins.

\section{Discussions}

These results suggest that tropical ulcers are associated with the presence of large numbers of unusual bacteria. While complete identification is not possible, the predominant organisms in acute untreated lesions correspond most closely in morphological appearance to Fusobacteria and some Bacteroides sp. Gram positive bacteria were scantily distributed at the sites of tissue destruction. The ultrastructural appearances of spirochaetes found were likely to be those of Treponema species because of their flagellar structure, length, and periodicity. ${ }^{6}$ Healing, acanthosis, and loss of dermal oedema as well as absence of bacteria are associated with antibiotic treatment. Patients receiving penicillin or metronidazole showed complete absence of fusobacteria and spirochaetes.

These features differ from those seen in mycobacterial ulcers, particularly Buruli ulcer caused by Mycobacterium ulcerans. In this condition epidermal proliferation in the edge of the ulcer does not occur and the amount of collagen necrosis is more extensive. Despite the pronounced necrosis the inflammatory infiltrate is minimal. ${ }^{7}$ Special stains show the presence of causative organisms in large numbers. Ecthyma is a bacterial infection in the dermis causing dermal necrosis with a severe inflammatory infiltrate of round cells. The surface is usually covered by a thick crust, mostly as a result of the exudation of serous fluid. The epidermis does not show the hyperplasia seen in tropical ulcer. ${ }^{7}$ Multiple 
Gram positive cocci can be shown by Gram stain in the upper dermis.

There was no evidence to implicate vasculitis or a thrombotic process in the pathogenesis of tropical ulcers from the material examined. All the vascular changes were likely to have resulted from the healing process and were typical of those seen in other chronic ulcers. No spirochaetes were identified in arterioles or arteries, suggesting that spirochaete associated endarteritis was not a factor. Indeed, endarteritis was only seen in one patient. The destruction of collagen and disruption of fibroblasts and inflammatory cells in the vicinity of large numbers of invading bacteria, however, suggest that the bacteria are cytotoxic. This is confirmed by recent studies showing that Fusobacteria but not Bacteroides species from patients with ulcers are toxic to cell lines such as Vero cells in vitro. ${ }^{8}$

We acknowledge the kind assistance of the Wellcome Trust who supported Dr Adriaans's work; the Royal College of Physicians of London and the Sir Ratanji Dalal Scholarship Committee (Royal College of Surgeons, London) for their generous help with Dr Robinson's studies. We also thank the many colleagues in Zambia, Gambia, southern India and
Adriaans, Hay, Lucas, Robinson

Papua New Guinea who helped with this investigation.

References

1 Robinson DC, Hay R. Tropical ulcer in Zambia. Trans Roy Soc Trop Med Hyg 1985;80:132.

2 Adriaans B, Hay R, Drasar B, et al. The infectious aetiology of tropical ulcer. A study of the role of anaerobic bacteria. $B r J$ Dermatol 1987;116:31.

3 Lowenthal LJ. Tropical phagedenic ulcer: a review. Int Rev Trop Med 1963;2:267.

4 Ngu VA. Tropical ulcers. Br Med J 1967;i:283.

5 Holdeman V, Cato E, Moore W. Anaerobe laboratory manual. 4th Ed. Blackburg, Virginia; VPI Laboratory, 1977:144.

6 Hovind-Hougen K. Determination by means of electron microscopy of morphological criteria of value for the classification of some spirochaetes in particular treponemes. Acta pathologica Microbiologica (Scand) (Suppl B,) 1976;255:16.

7 Pinkus H, Mehregan A. A guide to dermatohistopathology. 3rd Ed. New York: Appleton-Century-Crofts, 1981.

8 Grenier D, Maynard D. Cytotoxic effects of culture supernatants of oral bacteria and various organic acids on Vero cells. Can $J$ W Microbiol 1985;31:302.

Requests for reprints to: Dr Beverley Adriaans, Medical Microbiology, London School of Hygiene and Tropical Medicine, Keppel Street, London WCIE 7HT, England. 